\title{
ESTIMATIVA DE GRAUS-DIA ACUMULADOS NO CICLO DE FRUTIFICAÇÃO DE CULTIVARES DE ABACATEIROS (Persea americana, Miller).*
}

\author{
Antonio A. Lucchesi ** \\ Heitor W.S. Montenegro **** \\ Nilson A. Villa Nova $* * * * *$ \\ Antonio C. Florencio *****
}

RESUMO

De acordo com os dados climáticos da região de cultivo, utilizando-se da somatória de graus-dia acumulados, acima de um mínimo denominado temperatura base, pode-se estimar períodos de desenvolvimento, época de maturação e mesmo épocas de se processar controles fitossanitários. Com essas finalidades, estudou-se o comportamento, com relação ao ciclo de frutificação, das cultivares Pollock, Collinson, Prince $e$ Wagner, em pomares de três regiões climaticamente diferentes do Estado de São Paulo, Novo Horizonte, Limeira e Itapetininga.

Para o cálculo da somatória de graus-dia acumulados no ciclo de frutificação dessas cultivares, em cada região, utilizou-se da metodologia de VILLA NOVA et alii (1972) em função das temperaturas máximas e mínimas mensais, dados meteorológicos esses, obtidos na Seção de Climatologia Agrícola do Instituto Agronômico do Estado de São Paulo.

Através dos estudos realizados verificou-se que uma determinada cultivar, mesmo sendo cultivada em regióes climaticamente diferentes, requer uma mesma quantidade de graus-dia para completar o seu ciclo de frutificação.

Verificou-se que a cultivar Pollock necessita em média, 2513 graus-dia; a Collinson de 3767; a Prince de 4138 e a Wagner de 4480 . Dependendo da raça de origem dos abacateiros, pode-se determinar uma faixa de graus-dia acumulados para as diferentes cultivares completarem o ciclo de frutificação. Assim, as da raça Antilhana, consideradas precoces, necessitam de 2164 a 3554; as da Guatemalense consideradas tardias requerem 3729 a 4708 e as híbridas (Antilhana X Guatemalense), de 3278 a 3790 graus-dia acumulados.

* Entregue para publicação em 09-11-1977.

* * Departamento de Botânica da ESALQ.

*** Departamento de Agricultura e Horticultura ta ESALQ.

**** Departamento de Física e Meteorologia da ESALQ.

***** Acadêmico, estagiário do Departamento de Botânica da ESALQ. 


\section{INTRODUÇÃO}

A planta, necessita, para todas as suas fases, desde a germinação, crescimento, desenvolvimento até a maturação final de determinadas "unidades térmicas".

O total de "unidades térmicas" que cada planta necessita, pode, conforme o clima da região, provocar uma maturação mais precoce ou mesmo retardá-la.

Além disso, através da somatória dessas "unidades térmicas", em cada região de cultivo, pode-se estimar os períodos de desenvolvimento de cada fase da planta até a época de maturação final e a consequente época de colheita, e até mesmo prever-se controles fitossanitários.

Para a planta completar o seu ciclo calcula-se esse período através da soma dos excedentes de graus de temperatura acima de um mínimo, abaixo do qual o seu desenvolvimento é praticamente nulo. A essa temperatura mínima dá-se o nome de temperatura base (TB).

Existem, para muitas culturas, somatórias de "unidades térmicas", também denominadas graus-dia, e que são utilizadas para o planejamento agrícola de uma região.

No caso da cultura do abacateiro, praticamente não se encontra dados relacionados a esse assunto, mesmo porque as variedades cultivadas de abateiro originam-se de três regiões distintas climaticamente, além dos híbridos, o que dificulta o estudo dessa frutífera, com relação a esse assunto.

O presetne trabalho tem a finalidade de trazer informações para sanar, em parte, essa lacuna.

\section{REVISÃO DA LITERATURA}

DAMARIO e PASCALE (1971) citam que a temperatura do ar é o elemento climático mais importante no crescimento e desenvolvimento dos vegetais determinando, em grande parte a distribuição geográfica das comunidades botânicas naturais e a dos cultivos agrícolas. A ação positiva desse elemento bioclimático no desenvolvimento se mede mediante a acumulação diária de temperaturas a partir de um ponto de crescimento mínimo, variável em cada espécie vegetal, procedimento que tem recebido distintas denominações: soma de temperaturas, de unidades térmicas, de unïdades de calor ou de graus-dia, sinônimos que indicam a acumulação residual diária a partir de uma temperatura base, somatória que se realiza para o ciclo total ou para os subperíodos que o integram. 
ALLEN (1976) comenta que a somatória de graus-dia pode ser utilizada para se prever períodos de desenvolvimento de plantas e suas pragas.

Com relação a temperatura base (TB), ou seja a temperatura do ambiente em que a planta vive, abaixo da qual o seu desenvolvimento é praticamente nulo, HOPP et alii (1968) afirmam que de maneira geral culturas de verão (feijão, milho, tomate, algodão, melão) tem TB entre $10 \mathrm{e} 13^{\circ} \mathrm{C}$ enquanto que culturas de inverno (trigo, aveia, ervilha, espinafre) possuem TB entre 3 e $6^{\circ} \mathrm{C}$. VALLI (1965) cita que para a banana e amendoim, a 'T'B é de $18^{\circ} \mathrm{C}$. NEWMAN e BLAIR (1968) citam $10^{\circ} \mathrm{C}$ para o milho; e KISH $(1967) 10^{\circ} \mathrm{C}$ para a vagem.

MOTA et alïi resumem em uma tabela informações de diferentes autores que fornecem a temperatura base, em graus Celsius, para várias culturas: abóbora e citrus 12,8; alface e linho 4,4; aspargo 5,6; aveia, cevada e trigo de primavera 4,4 a 6,1 ; arroz 10,0 a 15,6 ; batatinha 6,1; ervilha 5,6; espinafre 2,2; feijão 10,0 a 11,7 ; melão e videira 10,0 ; milho 10,0 a 12,8 ; milho doce 9,4 a 10,0 ; pimenta 15,6 ; soja 15,0 ; tomate a 11,7 .

Segundo VILLA NOVA e SANTOS (1976) a experiência tem demonstrado que durante o ciclo de uma cultura a somatória dos graus-dia é acentuadamente constante, desde que não existam fatores limitantes naturais do desenvolvimento, tais como, deficiências de água, de nutrição, pragas, moléstias, etc. Esses autores citam exemplos como no Rio Grande do Sul, o milho precoce e o milho tardio $\left(\mathrm{TB}=10^{\circ} \mathrm{C}\right)$ possuem a somatória de graus-dia, desde a semeadura até o espigamento, respectivamente de 900 e 1100 . Esses dados dependem, é lógico, da cultivar e das características edafo-climáticas do local de plantio.

CARTER e PONELEIT (1973) estudando cultivares de milho, nos Estados Unidos da América verificaram que a somatória de graus-dia requerida para a maturação variava de 1337 a 1808.

MC MAHON e LOW (1972) ao estudar cultivares de algodão na Austrália verificaram que a somatória de graus-dia necessária até a colheita variava de 2250 a 3000 .

Para o abacateiro, poucos são os trabalhos encontrados, relacionados com esse assunto.

LUCCHESI e MONTENEGRO (1975) estudando o desenvolvimento de frutos do abacateiro, em três regiões ecologicamente diferentes do Estado de São Paulo constaram que houve uma dilatação no ciclo de frutificação (do florescimento a maturação) do abacate, a medida que o clima đa região torna-se mais frio. Relacionando-se as regióes estudadas, em uma determinada data, os frutos apresentavam diferen- 
tes graus de desenvolvimento fisiológico; mais adiantados na região mais quente e mais atrasados na região mais fria.

Segundo POPENDE (1924) todas as variedades cultivadas de abacateiros pertencem a mesma espécie botânica, e através de estudos botânicos, classificou-as, segundo os caracteres em comum, em três raças.

- Raça mexicana: plantas nativas de áreas elevadas do centro e do norte do México, com maturação do fruto no verão; sendo as mais resistentes ao frio;

- Raça guatemalense: plantas nativas de regióes elevadas da Guatemala e sul do México, cujo amadurecimento se dá no inverno e primavera; sendo menos resistentes ao frio; e

- Raça antilhana: plantas nativas das regiões baixas dos trópicos, com maturação do fruto ou verão e outono, sendo as mais susceptíveis ao frio.

Além das variedades cultivadas, provenientes das três raças existem os híbridos, que se originaram por polinização cruzada entre cultivares de raças diferentes (STAHL, 1933).

$\mathrm{Na}$ época de florescimento do abacateiro, segundo PRALORAN (1970), para que o fenômeno da dicogamia se processe sem perturbações a temperatura mínima não deve estar abaixo de 6,5 a $10,5^{\circ} \mathrm{C}$, para as três raças.

\section{MATERIAL E MÉTODOS}

Em três regiões ecologicamente diferentes do Estado de São Paulo (Novo Horizonte, Limeira e Itapetininga) foram realizadas observações na frutificação (do florescimento a maturação) de variedades de abacateiros cultivadas economicamente, quais sejam: Pollock (Fucks), Collinson, Prince e Wagner.

Em cada região, estudou-se o comportamento dessas variedades cultivadas, com a finalidade de se estimar os graus-dia acumulados no ciclo de frutificação.

$\mathrm{Na}$ região de Novo Horizonte foram observados abacateiros da Fazenda Santa Maria, de propriedade de Domingos Baraldo e Filhos, com 10 a 15 anos de idade. Na região de Limeira, pomares da Fazenda Citra, de propriedade de Dierberger Agrícola S.A., com abacateiros de 30 a 40 anos de idade; pomares da Chácara Paineira, de propriedade de Guilherme Ebeling, com abacateiros de 15 a 20 anos de idade; e pomares da Chácara Repouso, de propriedade de Miguel Guidotti, com abacateiros de 15 a 20 anos de idade. Na região de Itapetininga foram observados abacateiros dos pomares da Chácara Bela Vista, de proprie- 
dade de Paulo Tutomu Ito; da Granja Kato, de Shinjiro Kato; e da Granja Hanayama, de propriedade de Kinichi Hanayama; abacateiros esses, todos com a idade de 8 a 10 anos.

Os solos das regiões estudadas são: Novo Horizonte-podzólico vermelho-amarelo "intergrade" para latossol vermelho-amarelo; Limeira-podzólico de Lins e Marília, variação Lins; e Itapetininga-latossol vermelho-escuro orto.

Os parâmetros geográficos das regiões estudadas são:

Novo Horizonte: altitude de 453m; 21 28'02" de latitude sul e 49'13'17'" de longitude W.Gr.;

Limeira: altitude de 542m; 2233'49" de latitude sul e $47^{\circ} 24^{\prime} 15^{\prime \prime}$ de longitude W.Gr.; e

Itapetininga: altitude de $670 \mathrm{~m} ; 2^{\circ} 35^{\prime} 09^{\prime \prime}$ de latitude sul e $48^{\circ} 02$ '51" de longitude W.Gr.

As infcrmações meteorológicas utilizadas no presente trabalho foram obtidas na Seção de Climatologia Agrícola do Instituto Agronômico do Estado de São Paulo, Campinas (SP) .

Os dados meteorológicos utilizados para os pomares de Novo Horizonte foram tomados de uma estação meteorológica oficial de Pindorama, distando $50 \mathrm{Km}$ de Novo Horizonte. Pindorama situa-se a $562 \mathrm{~m}$ de altitude, $21^{\circ} 10^{\prime}$ de latitude e $48^{\circ} 54^{\prime}$ de longitude.

Da mesma forma, por não ser possível obter dado meteorológico de Itapetininga, foram tomados os de Tatuí, $35 \mathrm{Km}$ de Itapetininga,

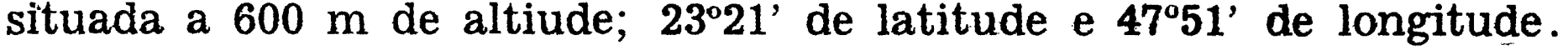

Para o cálculo da somatória de graus-dia no ciclo da frutificação para cada variedade cultivada de abacateiro, e para cada região estudada, utilizou-se da metodologia de VILLA NOVA et alii (1972), em função das temperaturas máxima e mínima médias mensais, em cada região.

A temperatura base para o abacateiro, foi estimada em $10^{\circ} \mathrm{C}$, baseando-se em PRALORAN (1970).

Nos Quadros 1, 2 e 3 encontram-se os dados utilizados para a obtenção da somatória de graus-dia (G.D.), em função da temperatura (TM) e da temperatura mínima (Tm), médias mensais, para cada região estudada. 
QUADRO 1 - Dados relativos à região de Novo Horizonte (os dados climáticos são média de 22 anos).

\begin{tabular}{lcccccccccccc}
\hline Meses & Jun & Jul & Ago & Set & Out & Nov & Dez & Jan & Fev & Mar & Abr & Mai \\
\hline$\overline{\mathrm{TM}}$ & 25,9 & 26,3 & 28,7 & 30,5 & 30,0 & 30,0 & 29,8 & 29,8 & 29,7 & 29,8 & 28,6 & 26,8 \\
$\overline{\mathrm{Tm}}$ & 12,7 & 12,3 & 13,7 & 15,9 & 17,4 & 17,9 & 18,8 & 19,4 & 19,5 & 18,8 & 16,1 & 13,8 \\
$\overline{\mathrm{G} . D}$ & 9,3 & 9,3 & 11,2 & 13,2 & 13,7 & 14,0 & 14,3 & 14,6 & 14,6 & 14,3 & 12,4 & 10,3 \\
dias & 30 & 31 & 31 & 30 & 31 & 30 & 31 & 31 & 28 & 31 & 30 & 31 \\
$\mathbf{\Sigma} \overline{\mathrm{G} . D}$ & 279 & 288 & 347 & 396 & 425 & 420 & 443 & 453 & 409 & 443 & 372 & 319 \\
\hline
\end{tabular}

QUADRO 2 - Dados relativos à região de Limeira (os dados climáticos são média de 33 anos).

\begin{tabular}{lcccccccccccc}
\hline Meses & Jun & Jul & Ago & Set & Out & Nov & Dez & Jan & Fev & Mar & Abr & Mai \\
\hline$\overline{\mathrm{TM}}$ & 24,6 & 24,8 & 27,3 & 28,4 & 28,4 & 28,7 & 28,9 & 29,3 & 29,1 & 29,1 & 27,6 & 25,5 \\
$\overline{\mathrm{Tm}}$ & 11,0 & 10,3 & 11,7 & 13,2 & 14,8 & 15,6 & 16,7 & 17,8 & 17,9 & 17,1 & 14,4 & 12,2 \\
$\overline{\mathrm{G} . \mathrm{D}}$. & 7,8 & 7,6 & 9,5 & 10,8 & 11,6 & 12,2 & 12,8 & 13,6 & 13,5 & 13,1 & 11,0 & 8,9 \\
dias & 30 & 31 & 31 & 30 & 31 & 30 & 31 & 31 & 28 & 31 & 30 & 31 \\
$\mathbf{\mathrm { S }} \overline{\mathrm{G} . \mathrm{D}}$. & 234 & 236 & 295 & 324 & 360 & 366 & 397 & 422 & 378 & 406 & 330 & 276 \\
\hline
\end{tabular}

QUADRO 3 - Dados relativos à região de Itapetininga (os dados climáticos são miédia de 19 anos).

\begin{tabular}{lcccccccccccc}
\hline Meses & Jun ... Ju1 & Ago & Set & Out & Nov & Dez & Jan & Fev & Mar & Abr & Mai \\
\hline $\overrightarrow{\text { TM }}$ & 24,2 & 24,5 & 24,8 & 27,8 & 27,7 & 28,9 & 29,4 & 30,0 & 29,7 & 29,5 & 27,5 & 125,1 \\
$\overline{\text { Tm }}$ & 8,8 & 8,3 & 9,6 & 12,2 & 14,2 & 14,9 & 16,7 & 17,6 & 17,7 & 16,6 & 13,8 & 10,3 \\
$\overline{\text { G.D }}$ & 6,5 & 6,5 & 7,2 & 10,0 & 11,0 & 11,9 & 13,1 & 13,8 & 13,7 & 13,1 & 10,7 & 7,7 \\
dias & 30 & 31 & 31 & 30 & 31 & 30 & 31 & 31 & 28 & 31 & 30 & 31 \\
$\Sigma \overline{\text { G.D.D. }}$ & 195 & 201 & 223 & 300 & 341 & 357 & 406 & 428 & 384 & 406 & 321 & 239 \\
\hline
\end{tabular}

\section{RESULTADOS E DISCUSSÃO}

O QUADRO 4 apresenta o valor de graus-dia acumulados, acima da temperatura base, desde a época de maior intensidade de florescimento até a época de maturação, em cada região produtora, e para cada cultivar estudada. 
QUADRO 4: Graus-dia acumulados na frutificação de cada cultivar de abacateiro.

\begin{tabular}{cllll}
\hline Cultivares & $\begin{array}{c}\text { Região } \\
\text { Produtora }\end{array}$ & $\begin{array}{l}\text { Época de maior } \\
\text { intensidade de } \\
\text { florescimento }\end{array}$ & $\begin{array}{l}\text { Epoca de } \\
\text { Maturação }\end{array}$ & $\begin{array}{l}\text { Graus-dia } \\
\text { Acumulados }\end{array}$ \\
\hline $\begin{array}{c}\text { Pollock* } \\
\text { (Antilhana) }\end{array}$ & $\begin{array}{l}\text { Novo Horizonte } \\
\text { Limeira }\end{array}$ & $\begin{array}{l}\text { agosto } \\
\text { agosto }\end{array}$ & $\begin{array}{l}\text { janeiro } \\
\text { feverciro }\end{array}$ & 2484 \\
\hline Collinson & Novo Horizonte & agosto & abril & 3742 \\
(Antilhana X & Limeira & agosto & junho & 3790 \\
Guatemalense) & Itapctininga & setembro & agosto & 3801 \\
\hline & Novo Horizonte & setembro & julho & 4247 \\
Prince & Limeira & setembro & agosto & 4024 \\
(Guatemalense) & Itapctininga & outubro & outubro & 4142 \\
\hline & Novo Horizonte & setembro & agosto & 4594 \\
WAGNER & Limeira & setembro & setembro & 4348 \\
(Guatemalense) & Itaptininga & outubro & novembro & 4499 \\
\hline
\end{tabular}

* Não foi encontrado pomar na região de Itapetininga com a cultivar Pollock.

Observa-se, através dos dados apresentados no QUADRO 4, que uma determinada cultivar, possui praticamente a mesma quantidade de graus-dias para sua frutificação, mesmo estando ambientada em regiões climaticamente diferentes.

Observa-se ainda, que cultivar de raça Antilhana, como no caso a Pollock, devido a sua região de origem, das regiões baixas dos trópicos, requer uma somatória menor em graus-dia, que as da raça Guatemalense, como no caso Prince e Wagner, originados das regiões elevadas da Guatemala e sul do México.

Tal fato concorda com o trabalho de LUCCHESI e MONTENEGRO (1975) que verificaram que há uma dilatação no ciclo de frutificação do abacate, a medida que o clima da região torna-se mais frio.

Analisando-se os dados obtidos, e relacionando-os com informações conhecidas das principais variedades de abacateiros cultivadas economicamente, pode-se estimar as somatórias de graus-dia dessas cultivares, de acordo com a raça de origem.

Assim, cultivares da raça Antilhana, consideradas precoces, como: Pollock, Simmonds, Princesa, Waldin, Barker e Baroneza, requerem para completar o ciclo de frutificação, de 2164 a 3554 graus-dias, de acordo com suas adaptações às regiões onde estão sendo cultivadas.

As da raça Guatemalense consideradas tardias, como: Wagner, Linda, Prince, Taylor, Itzamna e Rincon, requerem de 3729 a 4708 graus-dia acumulados para completarem o ciclo de frutificação. 
No caso das cultivares híbridas de Antilhana com Guatemalense, como: Collinson, Fortuna, Quintal e Nimlioh, requerem de 3278 a 3790 graus-dia para completarem o ciclo de frutificação.

Essas informações podem fornecer ao agricultor com conhecimento dos principais dados climáticos da região, uma boa orientação com respeito a época de maturação das variedades de abacate por ele cultivadas, e mesmo informações sobre melhores épocas de se processar o controle fitossanitário.

\section{CONCLUSÕES}

Dos estudos realizados chegou-se às seguintes conclusões:

1. a) Constatou-se que uma determinada cultivar de abacateiro, mesmo sendo cultivada em regiões climaticamente diferentes, requer uma mesma quantidade de graus-dia para completar o seu ciclo de frutificação (do florescimento à maturação.

2. ${ }^{\text {) }}$ Verificou-se que a cultivar Pollock (Fucks) necessita, em média, 2513 graus-dia; a Collinson de 3767; a Prince de 4138 e a Wagner de 4480 graus-dia para completar o ciclo de frutificação.

3. ${ }^{\text {) }}$ De acordo com a raça de origem das cultivares de abacateiros, pode-se determinar uma faixa de graus-dia acumulados para as diferentes cultivares completarem o ciclo de frutificação. Assim, as da raça Antilhana, consideradas precoces necessitam de 2164 a 3554; as da Guatemalense, consideradas tardias, requerem 3729 a 4708 e as hibridas (Antilhana $x$ Guatemalense), de 3278 a 3790 graus-dia acumulados.

\section{SUMMARY}

ESTIMATION OF DEGREE DAYS ACCUMULATED IN THE CYCLE OF FRUITING OF AVOCADO (Persea americana, Miller) CUTTIVARS.

According to the meterological data of the cultivation region, using the summation of accumulated degree days above a minimum called basic temperature, we can inake the estimation of growth periods, maturation moment, and moments to make pest and disease controls. With this subject it was studied the behaviour with relations to the cycle of fruiting of the Pollock, Collinson, Prince, and Wagner cultivars in three regions of São Paulo State: Novo Horizonte, Limeira, and Itapetininga. It was used the matmol of VILLA NOVA et alii (1972) to make these determinations.

It wasverified that certain specific cultivar growing in climatically different resions needs the same quantity of degree days to complete the cycle of fruiting. Is was hetermined that the cultivar Pollock needs about 2513 degree days; Collinson 3767; I'rince 4138 and Wagner 4480.

Depending of the race of origin of the avocados, we can determine a ranse of accumulated degree days for the different cultivars to complete the cycle of fruting.

So, the cultivars of the Antilhana race, considered premature, need 2164 to 3554 ; the Guatemaiense racc, considered slow, need 3729 to 4708 , and the hibrids (Antillana $x$ Guatemalense) need 3278 to 3790 degree days accumulated. 


\section{LITERATURA CITADA}

ALLEN, J.C., 1976. A modified sine wave nethod for calculating degree days. Environ. Entomol. 5(3):388-396.

CARTER, M.W. \& PONELEIT, C.G., 1973. Black layer maturity and filling period variation among inbred lincs of corn (Zea mays L.). Crop. Sci. 13(4):436-439.

DAMARIO, E.A. \& PASCALE, A.J., 1971 Estimación de sumas de temperaturas efectivas normales para estudios agroclimáticos. Rev. Fac. Agr. Vet. Univ. Buenos Aires 19(3): 109-124.

HOPP, R.J.; LAUTZENHEISER, R.E.; \& VARNEY, K.E.. 1968. Growwing degree days in Vermont. Vermont Agr. Exp. Sta. Bull. n. ${ }^{\circ} 659$, Burlington, 16 p.

KISH. A.J., 1967. Forecasting maturity date of snap beans in South Carolina. U.S. Dept. Comm. Tech. Mem. n. ${ }^{\circ} 20$, New York, 7 p.

LUCCHESI, A.A. \& MONTENEGRO, H.W.S., 1975. Influência ecológica no desenvolvimento do fruto e no teor de óleo na polpa do abacate (Persea americana Miller). Aanais da Esc. Sup. Agric. "Luiz de Queiroz", vol. 32.

MAC MAHON, J. \& LOW, A., 1972. Growing degree days as a measure of temperature effects on cotton. Cotton Grow Rev., Aust., 49(1):39-49.

MOTA, F.S.; ACOSTA, M.J.C.; ELLIS, J. \& VALENÇA, A.S., 1977. Disponibilidades térmicas para a agricultura nos Estados do Rio Grande do Sul, Santa Catarina, Paraná, São Paulo e Rio de Janeiro. Ciência e Cultura 29(9): 1006-1020.

NEWMAN, J.E. \& BLAIR, B.O., 1968. Growing degree days and dent corn maturity. Part II, Corn Production. Proc. Indiana, p. 19-31.

POPENOE, W., 1924. The avocado. In Manual of tropical and subtropical fruits. The Macmillan Company, New York, p. 9-78.

PRALORAN, J.C., 1970. Le climat des aires d'origine des avocatiers. Fruits 25(7-8): 543-557.

STAHL, AL., 1933 Changes in composition of Florida avocados in relation to maturity. University of Florida. Agricultural Experiment Station Gainesville, USA, Bull. 259, 61 p.

VALLI, V.J., 1965 Biometeorological factores as predictors of agronomic maturity of peanuts. Tech. Note n. 6 AGMET 2, U.S. Dept. Comm. Washington, $10 \mathrm{p}$.

VILLA NOVA, N.A.; PEDRO JR., M.J.; PEREIRA, A.R. \& OMETTO, J.C., 1972. Estimativa de graus-dia acumulados acima de qualquer temperatura base, em função das temperaturas máxima e mínima. Caderno de Ciências da Terra, Instituto de Geografia, USP, n. ${ }^{\circ} 30,8$ p.

VILLA NOVA, N.A. \& SANTOS, J.M., 1976. Unidades de calor (Graus-dia). Departamento de Física e Meteorologia, E.S.A. "Luiz de Queiroz", Piracicaba (SP), 6 p. (apostilado). 
